\title{
Light Ion Hohlraum Target Experiments On PBFA II and Nova
}

\author{
R. J. Leeper, J. E. Bailey, T. L. Barber, A. L. Carlson, G. A. \\ Chandler, D. L. Cook, M. S. Derzon, R. J. Dukart, D. E. Hebron, \\ D. J. Johnson, M. K. Matzen, T. A. Mehlhorn, A. R. Moats, T. J. \\ Nash, D. D. Noack, R. W. Olsen, R. E. Olson, J. L. Porter, J. P. \\ Quintenz, C. L. Ruiz, M. A. Stark, J. A. Torres, and D. F. Wenger
}

Sandia National Laboratories, Albuquerque, New Mexico 87185

\begin{abstract}
The goal of the National Inertial Confinement Fusion (ICF) Program in the United States is a target yield in the range of $200101000 \mathrm{MJ}$. To address this goal, the nearterm emphasis in the Light Ion Target Physics program is to design a credible high-gain target driven by ion beams. Based on this target design, we have identified ion beam spatial parameters, ion beam energy and power deposition, the conversion of ion-beam energy into soft $x$-ray thermal radiation, the conversion of ion-beam energy into hydrodynamic motion, radiation smoothing in low-density foams, and internal pulse shaping as the critical physics issues. These issues are currently being addressed in both ion- and laser-driven experiments.
\end{abstract}

\section{THE ION-DRIVEN TARGET CONCEPT}

The light ion ICF program is based upon an $\mathrm{x}$-ray driven or indirect-drive target concept. ' The light ion baseline target concept is shown schematically in Fig. 1. This target configuration is substantially different from either direct or indirect-drive laser target configurations. With a light ion beam driver, the fusion capsule is embedded in a region of low- $Z$ (low atomic number), low-density, $\mathrm{CH}$ foam. The foam is surrounded by a high- $Z$ radiation case or hohlraum. The incident high energy ions penetrate the high- $Z$ case and deposit most of their energy in the low- $Z$ foam. The hot, low- $Z$ plasma volumetrically radiates $x$-ray power at a rate that approximately balances the local ion deposition power. The majority of this $x$-ray power is absorbed and then re-emitted by the high- $Z$ hohlraum inner wall which is heated and ionized to produce a plasma radiator. The trapped radiation allows a relatively low average ion beam intensity to provide an $\dot{x}$-ray intensity at the capsule that is of the level required for an ICF implosion. The capsule is driven by a combination of $x$-rays emitted from the wall and from the foam. When the low- $Z$ foam is hot $(>70 \mathrm{eV})$, photons freely traverse the case-to-capsule distance, and the majority of the capsule drive originates at the high- $Z$ hohlraum wall. This is 


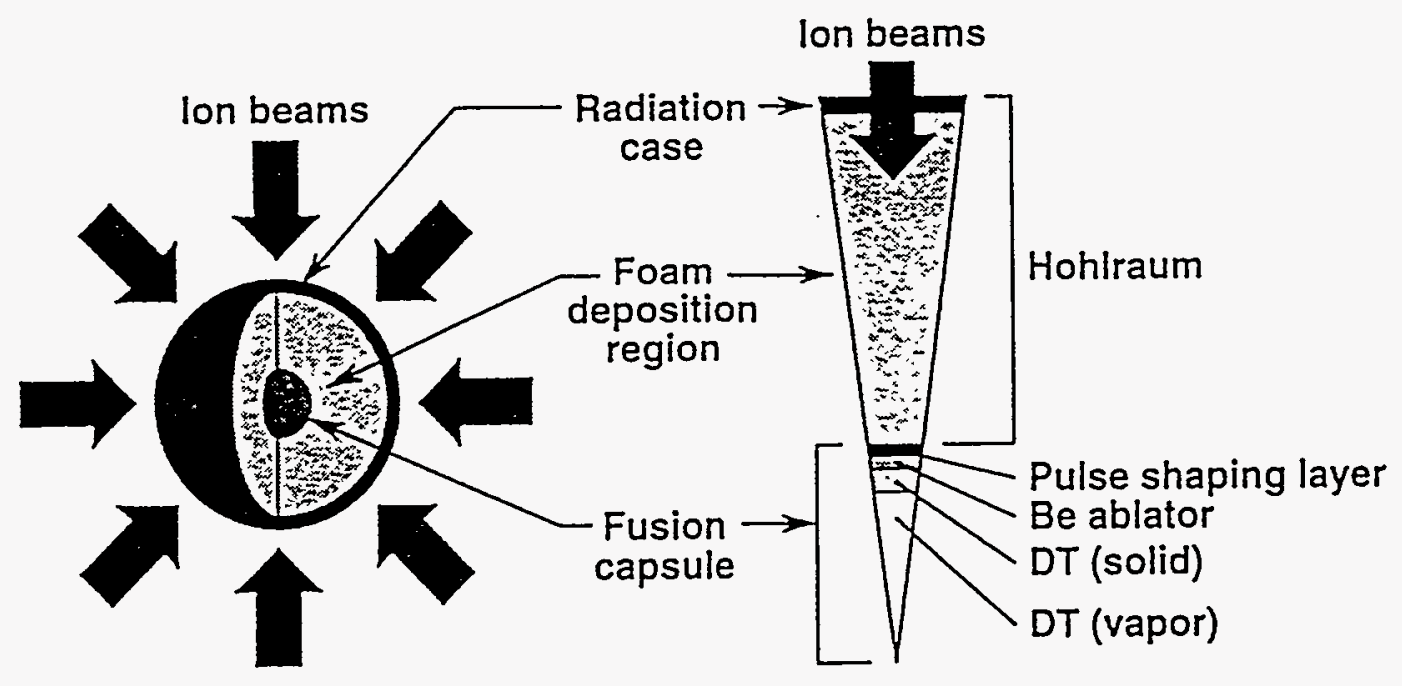

Fig. 1. Schematic of baseline light ion-driven ICF target.

possible due to the high albedo (the fraction of the absorbed $x$-rays that are reemitted is $>80 \%$ ) of the high- $Z$ wall plasma. By contrast, in an indirectly driven laser target, the hohlraum must have entrance holes to allow the laser energy to enter and strike the hohlraum interior wall. These entrance holes are a source of $x$ ray energy loss and are prone to closure at high power levels. Since the energy deposition and conversion physics is significantly different for ions and lasers, their potential failure mechanisms will be different. In this sense, in addition to their primary role as the preferred driver for high yield applications, ions represent a risk mitigation strategy in the near term ICF program as a backup to the mainline laser programs for ignition or breakeven experiments.

The goal of the experimental ion target effort on PBFA-II and Nova is to gain an understanding of the critical light ion beam hohlraum target issues at specific power deposition levels comparable to those required by the LMF hohlraum. Such experiments will provide key benchmarking of our ion deposition and radiation transport calculational capabilities.

\section{PBFA II EXPERIMENTAL RESULTS}

Several target experimental series have been carried out on the Sandia National Laboratories Particle Beam Fusion Accelerator II (PBFA II) facility. These experiments used a new comprehensive diagnostic suite to address critical target physics issues. ${ }^{2}$ These issues include ion beam spatial parameters, ion beam energy and power deposition, the conversion of ion-beam energy into soft $x$-ray thermal radiation, the conversion of ion-beam energy into hydrodynamic motion, radiation smoothing in low-density foams, and internal pulse shaping. Fig. 2 shows the three classes of targets that have been used in our experiments to study the conversion of ion-beam energy into radiation. The first target experiments on PBFA II employed intense proton beams to study thermal $x$-ray production in a 
cylindrical foam target shown in Fig. 2a. The proton parameters used in these experiments were a peak voltage of $6 \mathrm{MeV}$, a peak power density of $3 \mathrm{TW} / \mathrm{cm}^{2}$ averaged over the surface of a 6-mm- diameter spherical target, a full-width at halfmaximum (FWHM) of 13-15 ns, and a peak specific power density of $120 \mathrm{TW} / \mathrm{g}$. In an effort to increase the ion specific power density in our PBFA II experiments, $\mathrm{Li}$ beams were employed that increased the specific power density by over an order of magnitude. The hohlraum targets used in the $\mathrm{Li}$ experiments are shown in Figs. $2 b$ and $2 c$. The PBFA II Li beam had a peak voltage of $10 \mathrm{MeV}$, a peak power density of 1-1.5 TW/ $\mathrm{cm}^{2}$ averaged over a 6 -mm-diameter spherical target, a FWHM of 13-15 ns, and a peak specific power density of $1400 \mathrm{TW} / \mathrm{g}$. For both the proton and lithium experiments, the ion beam was accelerated from a cylindrical anode surface of radius $15.5 \mathrm{~cm}$ and was radially convergent onto targets located at the center of PBFA II from a full $360^{\circ}$ of azimuth with a vertical extent that was typically $\pm 17^{\circ}$. In either the proton or Li experiments, the beam passes through the Au walls of the target and, due to $\mathrm{dE} / \mathrm{dx}$ energy losses, is finally stopped in the 3-6 $\mathrm{mg} / \mathrm{cm}^{3} \mathrm{CH}$ foam region of the target. The rapid beam heating of the foam causes it to ionize and emit soft $x$-ray radiation. This soft $x$-ray emission in turn heats the gold walls of the target, which reradiate and create a hohlraum. With a specific power density of $1400 \mathrm{TW} / \mathrm{g}$, the lithium target experiments are in the physics regime of the foot of the $1000 \mathrm{TW} / \mathrm{g}$ power pulse required for a light-ion LMF.'

\section{Proton-Heated-Ion Hohlraum Experiments}

As described above, ion-driven hohlraums were first demonstrated with proton beams using the cylindrical target shown in Fig. 2a. The key ion-driven hohlraum characteristics observed with this target were foam heating of the hohlraum wall, foam tamping of the hohlraum wall, and a nominally Planckian radiation spectrum. ${ }^{3}$ To demonstrate the foam contribution to wall heating and its ability to tamp the hydrodynamic motion of the Au wall, experiments were conducted with and without a foam fill of $6 \mathrm{mg} / \mathrm{cm}^{3}$. The Au wall velocity with foam was measured to be $2.5 \pm 2 \mathrm{~cm} / \mu \mathrm{s}$. The Au velocity without foam was measured to be $\sim 14 \mathrm{~cm} / \mu \mathrm{s}$, clearly demonstrating the tamping action of the foam on the Au wall. The data also showed the radiated output of the foam-filled target was approximately four times the radiated output of the unfilled target, in good agreement with the calculated output. Further, there was good agreement between the proton experiments and the LASNEX modeling in the peak brightness temperature of $35 \pm 6 \mathrm{eV}$, the time to radiation peak of $15 \pm 4 \mathrm{~ns}$, and total radiation from aperture of $2.4 \pm 0.7 \mathrm{~kJ}$.

\section{Lithium-Beam-Heated Cone Hohlraum Experiments}

The first $\mathrm{Li}$ ion hohlraum experiments were carried out with the target shown in Fig. 2b. The conical shape was chosen so that diagnostics of the incident beam and the low energy $x$-ray emission have an adequate cross-section view of the target. The physics issues under study included $\mathrm{Li}$ coupling into the foam at $1400 \mathrm{TW} / \mathrm{g}$. optical transparency of the $\mathrm{CH}$ foam, the tamping of the Au wall by the foam, and 


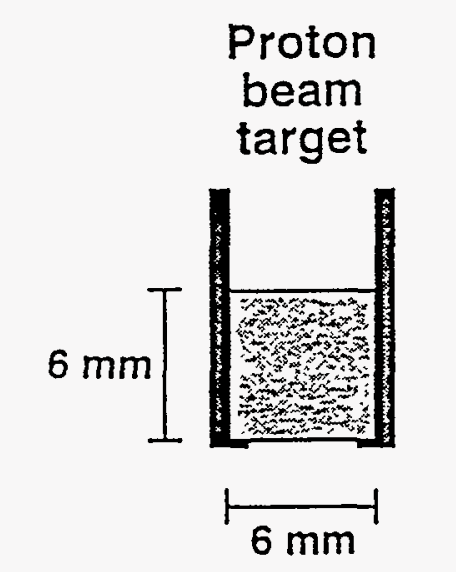

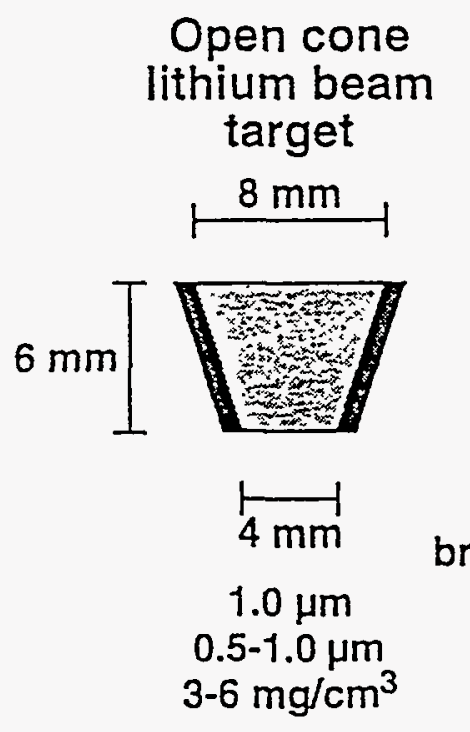

Fig. $2 b$
Cylindrical
protron beam target

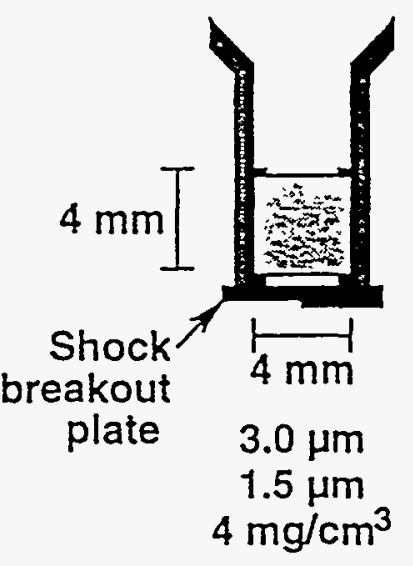

Fig. 2c

Fig. 2a

Fig. 2. Proton and lithium beam conical and cylindrical hohlraum targets designed for hohlraum physics studies on PBFA II.

the thermal $x$-ray emission. Viewed from the top, the spatial dependence of the soft $x$-ray emission is expected to show increased intensity in an annular profile corresponding to the cross-section of the gold cone and less emission from the central hole in the target (where there is no gold wall). This is observed in both the experiment and the modeling. Fig. 3a shows the calculated $x$-ray image for PBFA II shot 6000 . Fig. 3b shows a representative time-integrated soft $x$-ray camera image for this shot. The strong emission from the gold relative to the foam observed in this data and in the calculation is strong evidence that the foam is optically thin. Further evidence of the optical thinness of the foam is observed in the time-resolved images from the nine-frame $x$-ray imaging camera. ${ }^{2}$ Evidence of radiation smoothing and the hohlraum nature of this target system was also obtained from data that showed that a $25 \%$ asymmetry in the incident $\mathrm{Li}$ beam was smoothed to a $6 \%$ asymmetry in the thermal $x$-rays emitted below $280 \mathrm{eV}^{4}$ The average velocity of the gold wall during the power pulse was inferred from a 1-D timeresolved soft $x$-ray imaging diagnostic. The velocity was inferred to be less than the instrument resolution of $3 \mathrm{~cm} / \mu \mathrm{s}$. This result is consistent with results from other time-resolved imaging diagnostics as well as the modeling and simulations.

A peak brightness temperature of $58 \mathrm{eV}$ was obtained from the XRD diagnostic thermal $x$-ray flux. This result is consistent with the inferred radiation output and brightness temperature of the target obtained with a bolometer array diagnostic of $12.5 \mathrm{~kJ}$ into $2 \pi$ and $54 \mathrm{eV}$. 


\section{Top view of the open end of the cone as seen from $8^{\circ}$ off axis}

\section{Simulated data}

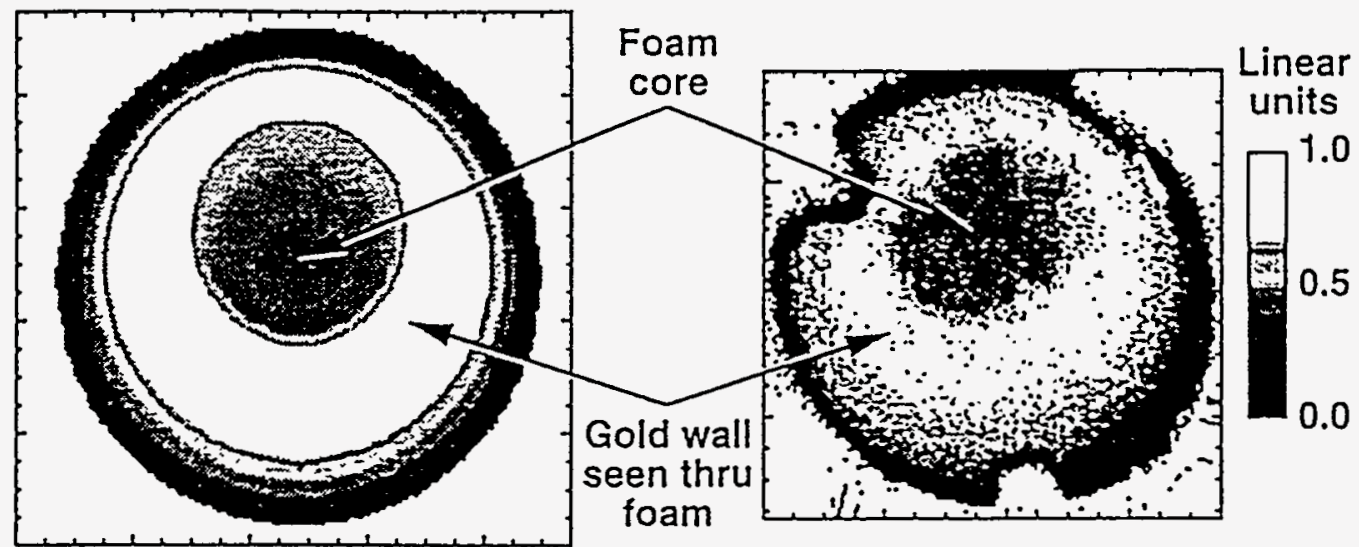

Fig. $3 a$

Fig. 3b

Fig. 3. Soft $\mathrm{X}$-ray $(200-280 \mathrm{eV})$ time-integrated images of $\mathrm{Li}$ ion beam cone. a) Calculated image of PBFA II shot 6000 . b) Measured image of PBFA II shot 6000 .

\section{Lithium-Beam-Heated Cylindrical Hohlraum Experiments}

For our latest lithium target series we fielded a new lithium beam target shown in Fig. 2c. The target was designed to maximize the radiation temperature by minimizing the hohlraum surface-to-volume ratio. The preliminary analysis of this shot series shows that we have demonstrated the highest radiation temperature yet achieved in an ion-beam-driven hohlraum, $63 \mathrm{eV}$. Ion beam properties derived from this data include beam symmetry, beam position, and beam intensity on the target. The radiation environment in the hohlraum was observed from the top of the target through a 1.5- or 3-mm diameter aperture. Time history temperature data from this series showed that the hohlraum is staying above $40 \mathrm{eV}$ for over $30 \mathrm{~ns}$.

\section{NOVA INTERNAL PULSE SHAPING EXPERIMENTS}

In our light ion target concept, the time-dependent pressure in the cryogenic DT fuel is controlled by using a two-step ion beam power pulse and a pulse shaping layer on the capsule.' In our most recent light ion target concept as shown in Fig. 1 , the outer two layers of the fusion capsule tailor the drive pressure history by combining an $\mathrm{x}$-ray filter effect with a change in the ablation pressure at the ablation front. This tailoring of the drive history is referred to as internal pulse shaping. The "filtering" is done by the outer pulse-shaping layer of the capsule by including higher- $Z$ materials than in the inner layers of the capsule. Early in time, the pulseshaping layer provides a relatively low pressure drive due to its relatively high albedo and relatively low ablation velocity. A sudden increase in pressure occurs 
when the ablation front moves into the inner, "main ablator". Later in time, when the ablation front is well into the main ablator region, the increasing temperature of the blow-off plasma from the pulse shaping layer results in a gradually decreasing filtering effect that can be used to control the rise in ablation pressure.

To validate the concept of internal pulse shaping, we have recently begun work on a series of proof-of-principal experiments on the Nova glass laser at Lawrence Livermore National Laboratory. We have made time-resolved measurements of shocks driven by radiation from burnthrough foils and pulse shaping layers attached to Nova hohlraums that were driven by a 2 ns square laser pulse to -200 $\mathrm{eV}$. The burnthrough radiation is monitored by a multichannel $\mathrm{K}$ - and L-edge filtered $x$-ray diode ${ }^{5}$ and a multichannel soft $x$-ray framing camera. ${ }^{6}$ The shocks generated in a witness plate are monitored with a Streaked Optical Pyrometer diagnostic. $^{7}$ Two dimensional radiation-hydrodynamics calculations have been used to predict the radiation drive conditions, the radiation burnthrough of thin foils, and the shock propagation in the witness plates. Ongoing experiments are aimed at generating a two-step shock structure with a square radiation drive pulse, where the materials in the pulse-shaping layer (filter) and ablator are contiguous to each other and are representative of materials used in a high yield target design.

\section{LIGHT ION TARGET SUMMARY}

The Sandia light ion ICF program is directed toward validating light ions as an efficient driver for high-yield ICF targets. With additional experiments on radiation transport and smoothing in a low-density foam environment to complement existing experiments on radiation production and internal pulse shaping, we will have demonstrated many of the critical physics issues for all high-yield ion targets, which are a key element of the eventual energy application of ICF.

\section{ACKNOWLEDGMENTS}

The authors would like to thank H. N. Kornblum, T. J. Orzechowski, D. Phillion, F. Ze, the Nova operations crew, and the PBFA II operations crew for invaluable technical assistance. This work supported by the U. S. Deparment of Energy under Contract No. DE-AC04-94AL85000.

\section{REFERENCES}

I. R. E. Olson et al., Proc. 15th IEEE Symp. on Fusion Engineering. p. 189 (1993)

2. R. J. Leeper et al., Rev. Sci. Instrum. 66, 511 (1995) and the references therein.

3. M. S. Derzon et al., Rev. Sci. Instrum. 63, 5068 (1992)

4. M. S. Derzon, submitted to Phys. Rev. Lett. (1995).

5. H. N. Kornblum et al., Rev. Sci. Instrum., 57, 2179 (1986).

6. F. Ze et al., Rev. Sci. Instrum., 63, 5124 (1992).

7. D. Phillion, Lawrence Livermore National Laboratory, private communication. 